\title{
THE INFLUENCE OF VARIOUS MICROENVIRONMENTAL FACTORS ON BIOMECHANICAL FEATURES OF DIFFERENT SUTURE MATERIALS USED IN HEPATO-PANCREATO-BILIARY SURGERY
}

\author{
Milan Radojković ${ }^{1}$, Miloš Stojković ${ }^{2}$, Ilija Golubović ${ }^{1}$, Dusan Sokolović ${ }^{3}$ \\ ${ }^{1}$ Surgery Department, Faculty of Medicine, University of Niš, Serbia \\ ${ }^{2}$ Laboratory of Intelligent Production Systems, Manufacturing Department, Faculty of Mechanical Engineering, \\ University of Niš, Serbia \\ ${ }^{3}$ Biochemistry Department, Faculty of Medicine, University of Niš, Serbia
}

\begin{abstract}
The key features of any suture material, such as its tensile strength, knot security, resorbability, handling characteristics and biological behavior must be taken into account during the selection process. These biomechanical features may be variable in different microenvironmental conditions in the human body in which the sutures are placed due to the influence of numerous local biohumoral factors. We have reviewed the data on the impact of pancreatic juice and bile, various $\mathrm{pH}$ conditions, chemotherapy and heat on different suture materials behavior. It is suggested that in pancreatic and biliary surgery polydioxanone sutures should be used. The review has also demonstrated that absorbable suture materials were more sensitive to $\mathrm{pH}$ than non-absorbable sutures. In addition, polyglyconate sutures were the strongest of all absorbable synthetic sutures when exposed to heat and cytotoxic drugs. This review provides a better basis for the selection of suture materials for specific applications.
\end{abstract}

Key words: tensile strength, suture materials, pancreatic juice, bile, $\mathrm{pH}$ condition, chemotherapy

\section{Introduction}

Surgical sutures are among the most commonly used medical devices. Their design and composition, along with the application technique, are essential for successful wound healing [1]. Although many issues are involved in the wound healing process special consideration should be taken with regard to the exposure of the suture materials to various microenvironmental biohumoral factors in the different tissues. These include different body fluids, $\mathrm{pH}$ conditions, local temperature etc.

Types of suture materials and their classifications are presented in Table 1. The most common classification of surgical suture materials is based in their resorbability and includes absorbable (AB) or non-absorbable (NAB) sutures. Also, based on their composition within these two categories, there are three types of materials used: natural unmodified, natural modified, and synthetic. In addition, based on their structure they are divided to monofilament and multifilament, coated or uncoated, with straight or curved needle. Furthermore, sutures can be classified according to their sizes as well as to the method of application, The performances of the suture material are adjusted to the specific tissue- and microenvironment-related conditions and demands, so the sutures are also classified for particular exclusive

Correspondence to: Milan Radojković, Ph.D

14/28 Sestara Baković Str., 18000 Niš, Serbia

Phone: +38 169716567

E-mail: mida71@open.telekom.rs

Received June $25^{\text {th }}, 2019$, Accepted January $19^{\text {th }}, 2020$ application in different types of surgery. The key features of any suture material, such as its tensile strength, knot security, resorbability, handling characteristics and biological behavior must be taken into account during the selection process [2].

Possible mechanisms underlying the changes of the suture features in different conditions will be discussed below, with an emphasis on pancreatic juice and bile, various $\mathrm{pH}$ conditions, chemotherapy and heat. Furthermore, these changes are also important considering that some of these materials are nowadays being widely investigated as possible constituents of some more complex prosthetic devices.

\section{Biomechanical properties of the suture material}

The biomechanical properties of the suture are of major concern to surgeons. The capacity of a suture to withstand the tension forces that lead to its stretching and rupture is defined as tensile strength. It is under the direct influence of numerous factors, most important being the material components, the cross-sectional area thickness and the stretching force. In terms of surgical practice, tensile strength is the time it takes for suture to lose $70 \%$ to $80 \%$ of its initial strength. Most available $\mathrm{AB}$ sutures halve their strength within 4 weeks. However, lactide-based copolymer $A B$ sutures can maintain the strength over the period of 2 months. For most of the $\mathrm{AB}$ sutures, required full absorption time ranges from several months to years [1]. It is important to notice that 
the loss of tensile strength and mass absorption are two separate processes. Although the suture loses its strength much faster than it is absorbed, it must support the wound long enough to enable healing after which it remains in the tissue as a foreign body for a much longer period [2].
In the early stages of wound apposition and healing, high tensile strength of sutures is important, while retention of strength is important in delayed wound healing situations [3]. Suture strength is influenced by various conditions prevailing in a particular part of the body. In many areas the changes of the fiber properties

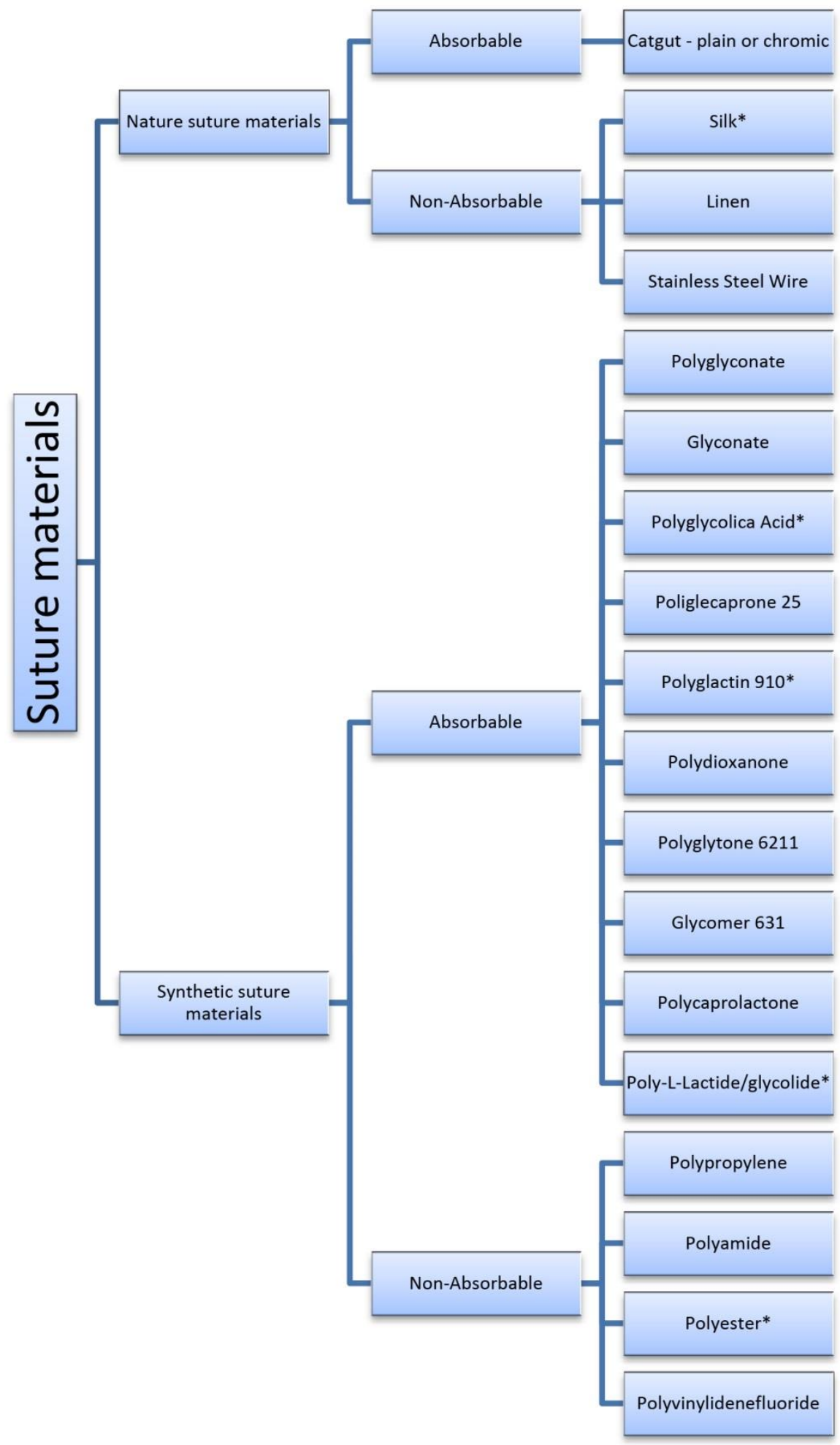

Fig. 1 Different types of suture materials with their classifications (natural and synthetic; absorbable and non-absorbable; mono-filament and multi-filament).

${ }^{*}$ Multi-filament (braided) sutures, whereas all other unmarked sutures (without*) are mono-filament. 
and weakening of the tensile strength occur due to the influence of various local biohumoral factors. Also, it is noted that the weakest point of a surgical suture is the knot, regardless of the suture material and the knot configuration [4, 5]. It is important to know the tensile strength of the suture and its changes in different $\mathrm{pH}$ environments $[6,7]$, under the influence of body fluids $[8,9]$, or due to the presence of bacterial adherence [10]. These changes may irreversibly alter the suture dynamics [11], knotting characteristics [12, 13] and biocompatibility [14], and eventually lead to impaired healing.

\section{Effect of pancreatic juice and bile on the tensile strength of suture materials}

It is assumed that pancreatico-jejunostomy has a higher failure rate compared to other surgical anastomoses. One of the reasons for that may be the exposure of the sutures to pancreatic juice. Specific effects of pancreatic juice and bile on the tensile strength of suture materials derive from the composition of these two body fluids. Bile contains water, electrolytes, bile salts, proteins, lipids, and bile pigments, and it is an alkaline fluid. Pancreatic fluid contains approximately fifteen enzymes or precursors of enzymes [8].

There is little data on the effects of pancreatic juice and bile on suture materials. The aim of the study by Muftuoglu et al. (2004) was to observe such effects on absorbable and non-absorbable suture materials [8]. The authors suggested that the application of catgut and silk should be avoided and for mucosal layer of pancreaticojejunostomy non-absorbable sutures should be used. This research also showed that PGA sutures and polyglactin 910 were not appropriate for use in pancreatic surgery. Furthermore, the authors proposed PDO sutures for application in pancreas surgery because of their resistance to pancreatic fluid and lesser associated inflammatory reaction [8]. Biliary and pancreatic surgery are associated with increased risk of fistula [8]. The nature of the suture used may have a role in the genesis of pancreatic fistula, in addition to multiple factors involved in the development of this complication [9]. The majority of absorbable suture materials are sensitive to proteolytic enzymes contained in pancreatic juice because they are made of proteins [9].

One of the earliest researches was the study by $\mathrm{Mi}$ zuma et al. investigating the changes of loop-tensile strength of various suture materials when exposed in vitro to pancreatic juice [9]. It was concluded that due to the exposure to pancreatic juices and consequent proteolysis, 24-48 hours after surgery, catgut could not maintain its tensile strength and could not hold the pancreatic duct and jejunum connected. Furthermore, their research demonstrated that catgut sutures were disintegrated even when almost all trypsin activity was inhibited and this was due to the presence of other proteolytic enzymes in the pancreatic juice that may be involved in the process of digestion. Also, non-activated pancreatic juice was capable of digesting the catgut because these pancreatic enzymes were active at least partially in the form in which they were secreted [9]. Interestingly, the finding that the addition of aprotinin has a protective effect might have practical significance [9].

According to the results of Mizuma et al., PGA sutures maintained their strength better than catgut, but not as well as silk or nylon. Also, because of their structure, polyglycolic sutures were digested by nonactivated pancreatic juice faster than by activated pancreatic juice [9]. Furthermore, in numerous studies it was concluded that synthetic suture materials were better for use in pancreas surgery $[15,16]$.

\section{Effect of various pH conditions on the ten- sile strength of suture materials}

It is very important for the surgeon to know how the strength of suture materials is influenced by the different chemical components and features of the body fluid in direct contact with the thread, particularly the $\mathrm{pH}$ level, after their implantation. In different circumstances, especially under pathologic conditions, the $\mathrm{pH}$ of body fluids may alter the biomechanical characteristics of the suture. Thus, a study of the $\mathrm{pH}$ effect on tensile strength of sutures will provide a better basis for the selection of suture materials for specific applications.

Under normal circumstances, the $\mathrm{pH}$ of gastric juice varies from 0.9 to 1.5 while pancreatic juice in the duodenum ranges from 7.5 to 8.2 and urinary $\mathrm{pH}$ ranges from 4.5 to 8.0. Chu's et al. categorized and summarized the effects of $\mathrm{pH}$ on eight different $\mathrm{AB}$ and $\mathrm{NAB}$ suture materials, based on an in vitro immersion study. The reported $\mathrm{pH}$ dependent degradation of sutures in this study deserves the attention of surgeons in their selection of these suture materials in various physiologic and pathologic conditions. Furthermore, in this study it was indicated that $\mathrm{AB}$ suture materials were more sensitive to $\mathrm{pH}$ than NAB suture materials [17].

Tomihata et al. showed that sutures containing glycolic acid as a comonomer were degraded faster in alkaline solution, whereas PDO sutures had a faster degradation in acidic solution [18]. In addition, Chu et al. (1983) demonstrated that both acidic and alkaline environments might accelerate the degradation of natural $\mathrm{AB}$ sutures, while only alkaline conditions had this undesirable effect on synthetic AB sutures. This observed $\mathrm{pH}$ dependence of PGA and polyglactin 910 sutures was consistent with the previous reports $[19,20]$, but it was contrary to Holm-Jensen and Agner's and Reed and Gilding's data [21, 22]. It is hypothesized that the coating materials and/or the level of crystallinity may contribute to this discrepancy. In the same investigation, within the NAB sutures, silk was the most sensitive in various $\mathrm{pH}$ conditions. Polyamide [multifilament) sutures were the next most sensitive to $\mathrm{pH}$ levels, while polyamide (mono-filament), polyester, and polypropylene sutures had very similar $\mathrm{pH}$ dependence [17].

Many authors [7,23] were consistent with results of Chu's et al. (1983) research indicating that accelerated 
loss of strength was present in both acidic and alkaline environments. Also, it is claimed that the suture implantation site, which is always presented with inflammation, is generally on the acidic side of the $\mathrm{pH}$ scale [17]. This may have a major importance in certain situations. For example, the transfixion of a bleeding ulcer in the environment with low $\mathrm{pH}$ due to the presence of $\mathrm{HCl}$ should not be performed with plain catgut sutures. NAB sutures that maintain their tensile strength for 4 weeks are the right choice in these situations. For closure of the intestines the choice should be the AB sutures in order to avoid the narrowing of the intestinal lumen and bacterial migration along the fibers [17].

Preservation of adequate strength in pancreatic juice is especially important. In Chu's study it was noted that polypropylene, polyester, and polyamide (mono-filament) were the NAB sutures of choice rather than silk sutures and polyamide (multifilament), while polyglactin 910 was the best among the $A B$ sutures. As for the urinary tract the $\mathrm{AB}$ suture is a better choice than NAB suture [17].

The study by Karabulut et al. (2010) aimed to investigate the effects of $\mathrm{pH}$ and in vivo and in vitro effects of different intra-abdominal organs and fluids on the retention of tensile strength of seven different suture materials. They summarized that polypropylene suture preserves its stability in all conditions and $\mathrm{pH}$ values. In addition, it is concluded that in urological surgery polyglytone 6211 and glycomer 631 should be used. In biliary surgery polyglactin 910 , polyglyconate or glycomer 631 had the best performances, while the use of polypropylene, as the material with highest durability, was disputed because it is an NAB suture and could cause biliary stone formation [7].

\section{Effect of chemotherapy and heat on the tensile strength of suture materials}

The absorption rate and therefore the tensile properties of $\mathrm{AB}$ sutures are dependent on their chemical properties. There is numerous evidence that free radicals [24], enzymes [25], temperature [26], and $\mathrm{pH}$ [7] may affect the hydrolysis rate of glycolic and lactic acids polymers used in $A B$ sutures structure. Because most of the chemical reactions increase with temperature rise, temperature is particularly important among these factors. The process of tissue repair depends on both the surgical technique and the properties of the sutures used, as well as on tissue integrity. It is noted that impaired wound healing induced by chemotherapy agents and/or hyperthermia after hyperthermic intraperitoneal chemotherapy (HIPEC) procedures has been associated with bowel complications observed [5].

Lapointe's et al. (2016) study described for the first time the impact of heated chemotherapy on biomechanical properties of the commonly used gastrointestinal sutures. Thus, the objective of this study was to compare tensile breaking force and elongation rate of six different $\mathrm{AB}$ sutures, when exposed to heat and cy- totoxic drugs: oxaliplatin and mitomycin-c, for a better understanding of their impact on gastrointestinal anastomosis. They concluded that tensile strength of all tested $\mathrm{AB}$ sutures was preserved for a minimum of 2 weeks. In addition, it was suggested that exposition to heat and chemotherapy did not significantly affect the biomechanical properties of tested sutures. However, this study did not analyze the long-term impact of heat and chemotherapy on sutures' properties. This study model was not sufficient for the final conclusion about the influence of suture choice on anastomotic leakage [5]. The selected heat model in this study included the temperatures of $37^{\circ} \mathrm{C}$ and $45^{\circ} \mathrm{C}$, because of the increased risk of small bowel toxicity when intra-abdominal temperature is over $45^{\circ} \mathrm{C}$ [15], while $37^{\circ} \mathrm{C}$ mimics the body temperature. So far there is no clear position on the choice of suture materials for the use in cytoreductive HIPEC procedures. Attitudes applied by surgeons nowadays are based on individual experience [5].

Beside the tensile strength, another important biomechanical feature of the suture materials is elongation rate which is determined by both elasticity and structural properties of the suture material. The elongation rate of suture materials determines the suture adjustment to both wound edema and wound contraction. As showed in Lapointe's et al. (2016) paper, monofilament sutures had a significantly higher elongation rate than multifilament sutures in all experimental conditions [5]. Furthermore, Tomihata et al. (2005) noted that monofilament sutures provide higher elongation rate than braided sutures [27]. This is explained by the structural characteristics of monofilament sutures, i.e. more homogenous tubular structure which contributes to the higher elongation rate [28,29]. It is important to point out that some studies reported no correlation between the suture tensile strength and its elongation rate [5,30]. Furthermore, increasing the size of suture material increases its tensile strength.

Lapointe et al. (2016) showed in their study that under basal condition polyglyconate was the strongest of all tested $\mathrm{AB}$ synthetic sutures while tensile strength was like other $\mathrm{AB}$ sutures investigated [5]. However, Pietrzak et al. showed that $\mathrm{AB}$ polymers used in suture composition were affected by temperature [26]. The authors analyzed the impact of temperature on hydrolysis of a PGA/polylactic acid copolymer. Their results showed that the rate of hydrolysis increased with the rise of temperature. The interesting observation in these data was that a variance of as little as $2^{\circ} \mathrm{C}$ from $37^{\circ} \mathrm{C}$ could affect the rate of hydrolysis for about $25-30 \%$ [26]. This adverse finding in relation to the results by Lapointe et al. may be explained by the difference in incubation time.

In addition to the temperature, it should be taken into account that the $\mathrm{pH}$ of the incubating solution is not a less important factor that can affect hydrolysis rate of the tested $\mathrm{AB}$ suture. However, the whole experimental model in the research conducted by Lapointe et al. would have different results if it was implemented under 
in vivo conditions [5], and therefore this is one of the main limitations of this study. Thus, these data must be interpreted with caution. Also, in an investigation by Pietzark et al. all animal testing needs to be interpreted with caution because most of them have a body temperature $1-3^{\circ} \mathrm{C}$ greater than that of humans [26]. It may be that these variations in body temperature may contribute to faster tensile strength loss in animals. More research is required to determine the efficacy of chemotherapy and heat on the tensile strength of suture materials.

One should bear in mind that this part of the review is based on a small amount of the published data. Further studies need to be carried out in order to validate these adverse findings and determine a definitive statement on the effect of chemotherapy and heat on the tensile strength of suture materials.

\section{Sutures and infection}

Although not the primary goal of this review, the influence of bacterial accumulation and infection on the biomechanical features of surgical sutures should be mentioned. It is now well known that surgical sutures may serve as a favorable substrate for bacterial colonization and growth [31]. This bacterial colonization induces a hypoxic environment and inhibits the activity of fibroblasts and granulocytes, thus leading to the impaired host-defense response. This is followed by the creation of biofilm consisting of bacteria encapsulated within a self-produced extracellular polymeric matrix composed of polysaccharides, proteins and nucleic acids which serves as a self-protection for bacteria [32, 33]. Susceptibility of suture materials for bacterial adherence depends on their biomechanical and chemical features. For example, it is widely accepted that braided sutures

\section{References}

1. Ingram D. Bioswellable amphiphilic copolymers, MSc thesis. Clemson University: South Carolina, USA, 2012.

2. Thomas WEG. Sutures, ligature materials and staples. Surgery 2002; 20(5):97-99.

3. Field JR, Stanley RM. Suture characteristics following incubation in synovial fluid or phosphate buffered saline. Injury 2004; 35(3): 243-248.

4. Silver E, Wu R, Grady J, Song L. Knot security - how is it affected by suture technique, material, size, and number of throws? J Oral Maxillofac Surg 2016; 74:1304-1312.

5. Lapointe S, Zhim F, Sidéris L, Drolet P, Célestin-Noël S, Dubé P. Effect of chemotherapy and heat on biomechanical properties of absorbable sutures. J Surg Res 2016; 200(1):59-65.

6. Chung E, McPherson N, Grant A. Tensile strength of absorbable suture materials: in vitro analysis of the effects of $\mathrm{pH}$ and bacteria. J Surg Educ 2009; 66(4):208-211.

7. Karabulut R, Sonmez K, Turkyilmaz Z, Bagbanci B, Basaklar $\mathrm{AC}$, Kale N. An in vitro and in vivo evaluation of tensile strength and durability of seven suture materials in various $\mathrm{pH}$ and different conditions: an experimental study in rats. Indian $\mathbf{J}$ Surg 2010; 72(5):386-390.

8. Muftuoglu MT, Ozkan E, Saglam A. Effect of human pancreatic juice and bile on the tensile strength of suture materials. Am J Surg 2004; 188(2):200-203. are more at risk of contamination due to their larger scabrous surface that facilitates bacterial adhesion in comparison with monofilament sutures. In turn, bacterial colonization and especially infection significantly diminish the quality features of sutures including, but not limited to their tensile strength. Therefore sutures may also be graded by the level of their infectibility. In terms of their resistance to infection, synthetic and monofilament sutures are suggested as superior than natural and multifilament ones. However, as this is the field of wide scientific interest numerous data on this topic would exceed the scope of this review.

\section{Conclusion}

The present review was designed to determine the effects of different conditions on tensile strength of suture materials. It is suggested that in pancreatic and biliary surgery PDO should be used; for mucosal layer of pancreatic anastomosis, non-absorbable sutures were sutures of choice. The review has also demonstrated that absorbable suture materials were more sensitive to $\mathrm{pH}$ than non-absorbable sutures. Both acidic and alkaline environments might accelerate the degradation of natural absorbable sutures while only alkaline conditions had this undesirable effect on synthetic absorbable sutures. This review has found that polyglyconate was the strongest of all tested absorbable synthetic sutures when exposed to heat and cytotoxic drugs. The current data highlight the importance of different conditions on biomechanical features of suture materials. The review is limited by the lack of published information on this issue. It is suggested that the association of these factors should be more intensely investigated in future studies.

9. Mizuma K, Lee PC, Howard JM. The disintegration of surgical sutures on exposure to pancreatic juice. Ann Surg 1977; 186(6): 718 .

10. Masini BD, Stinner DJ, Waterman SM, Wenke JC. Bacterial adherence to suture materials. J Surg Educ 2011; 68(2):101-104.

11. Visser JD. Dynamic strength of surgical suture materials. in: Winter GD, Leray JL, deGroot K. (eds): Evaluation of biomaterials. Wiley, London, 1980.

12. Tera H, Aberg C. Tensile strengths of twelve types of knot employed in surgery, using different suture materials. Acta Chir Scand 1975; 142(1):1-7.

13. Stone IK, Masterson BJ, Von Fraunhofer JA. Knot stability and tensile strength of an absorbable suture material. Surf Coat Tech 1986; 27(3):287-293.

14. Stillman RM. Wound closure: choosing optimal materials and methods. ER Reports 1981; 2: 41-44.

15. Merei I, Nahm C, Ofri A, Samra J, Clarke E, Anubhav M. A Comparative in vitro assessment of suture strength after exposure to pancreatic enzyme solution. JOP. J Pancreas (Online) 2019; 20(5):138-141.

16. Karaman K, Bal A, Aziret M, Ercan M, Bostanci EB, Akoglu M. Which suture material is optimal for pancreaticojejunostomy anastomosis? An in vitro study. J Invest Surg 2017;30(4):277-284.

17. Chu CC, Moncrief G. An in vitro evaluation of the stability of mechanical properties of surgical suture materials in various $\mathrm{pH}$ conditions. Ann Surg 1983; 198(2):223-228. 
18. Tomihata K, Suzuki M, Ikada Y. The $\mathrm{pH}$ dependence of monofilament sutures on hydrolytic degradation. J Biomed Mater Res 2001; 58(5):511-518.

19. Chu CC. A comparison of the effect of $\mathrm{pH}$ on the biodegradation of two synthetic absorbable sutures. Ann Surg 1982; 195(1):55.

20. Chu CC. The effect of $\mathrm{pH}$ on the in vitro degradation of poly (glycolide lactide) copolymer absorbable sutures. J Biomed Mater Res 1982; 16(2):117-124.

21. Holm-Jensen S, Agner E. Syntetisk absorberbart suturmateriale (PGA) sammenlignet med catgut. Ugeskrift Laeger 1974; 136(32):1785-1790.

22. Reed AM, Gilding DK. Biodegradable polymers for use in surgery - poly (glycolic)/poly (lactic acid) homo and copolymers: 2. In vitro degradation. Polymer 1981; 22(4):494-498.

23. Abellán D, Nart J, Pascual A, Cohen RE, Sanz-Moliner JD. Physical and mechanical evaluation of five suture materials on three knot configurations: an in vitro study. Polymers 2016; 8(4): 147.

24. Lee KH, Chu CC. The role of superoxide ions in the degradation of synthetic absorbable sutures. J Biomed Mater Res 2000; 49(1): 25-35.

25. Williams DF, Mort E. Enzyme-accelerated hydrolysis of polyglycolic acid. J Bioeng 1977; 1(3):231-238.
26. Pietrzak WS, Kumar M, Eppley BL. The influence of temperature on the degradation rate of LactoSorb copolymer. J Craniofac Surg 2003; 14(2):176-183.

27. Tomihata K., Suzuki M, Tomita N. Handling characteristics of poly (L-lactide-co- $\varepsilon$-caprolactone) monofilament suture. Biomed Mater Eng 2005, 15(5):381-391.

28. Kim JC, Lee YK, Lim BS, Rhee SH, Yang HC. Comparison of tensile and knot security properties of surgical sutures. J Mater Sci Mater Med 2007; 18(12):2363-2369.

29. Türker M, Kılıçoğlu Ö, Salduz A, Bozdağ E, Sünbüloğlu E. Loop security and tensile properties of polyblend and traditional suture materials. Knee Surg Sports Traumatol Arthrosc 2011; 19(2):296-302.

30. Freudenberg S, Rewerk S, Kaess M, Weiss C, Dorn-Beinecke A, Post S. Biodegradation of absorbable sutures in body fluids and $\mathrm{pH}$ buffers. Eur Surg Res 2004; 36(6):376-385.

31. Henry-Stanley MJ, Hess DJ, Barnes AM, Dunny GM, Wells CL. Bacterial contamination of surgical suture resembles a biofilm. Surg Infect 2010;11:433-439.

32. Hall-Stoodley L, Costerton JW, Stoodley P. Bacterial biofilms: from the natural environment to infectious diseases. Nat Rev Microbiol 2004;2:95-108.

33. Fux C, Costerton J, Stewart P, Stoodley P. Survival strategies of infectious biofilms. Trends Microbiol 2005;13:34-40. 